\title{
A Comparison of 3D Sensors for Wheeled Mobile Robots
}

\author{
Gerald Rauscher, Daniel Dube and Andreas Zell \\ Chair of Cognitive Systems, University of Tuebingen, Sand 1, 72076 Tuebingen, \\ Germany $\{$ g.rauscher, daniel.dube, andreas.zell\}@uni-tuebingen.de
}

\begin{abstract}
D Sensors are used for many different applications, e.g. scene reconstruction, object detection and mobile robots, etc. Several studies on usability and accuracy have been done for different sensors. However, all these studies have used different settings for the different sensors. For this reason we compare five 3D sensors, including the structured light sensors Microsoft Kinect and ASUS Xtion Pro Live and the time of flight sensors Fotonic E70P, IFM O3D200 and Nippon Signal FX6, using the same settings. The sensor noise, absolute error and point detection rates are compared for different depth values, environmental illumination and different surfaces. Also simple models of the noise depending on the measured depth are proposed. It is found that the structured light sensors are very accurate for close ranges. The time of flight sensors have more noise, but the noise does not increase as strongly with the measured distance. Further, it is found that these sensors can be used for outdoor applications.
\end{abstract}

\section{Introduction}

To enable mobile robots to move autonomously in a variety of unknown scenarios, 3D information about the environment is needed. In contrast to stereo vision, 3D sensors directly provide 3D data without extra computational efforts. The evaluated sensors use two different ways to measure the distances by emitting light: structured light or time of flight (ToF). Therefore, the sensors have different characteristics. Several studies of accuracy and usability of 3D sensors for mobile robots have been published [1-13]. The studies show that 3D sensors can be used for autonomous robot applications. However, it is difficult to compare the results, because most studies use individual settings i.e. different test objects and different evaluations. In this paper we compare the sensors Microsoft Kinect, ASUS Xtion Pro Live, Fotonic E70P, IFM O3D200 and Nippon Signal FX6, (the predecessor of the FX8). We propose repeatable methods to get empirical results about precision, accuracy and reliability of the sensor measurements under different conditions, like distance, lighting conditions, object size and surface. With these results it is easy to select an appropriate sensor for a certain application. For probabilistic approaches in robotics it is crucial to know the uncertainty of single measurements. Therefore we present a distance depending model of the expected standard deviation for each sensor. 


\section{Related Work}

3D Sensors are used for architectural and archaeological surveys, object modeling, 3D scene reconstruction [12], plant phenotyping [5] and mobile robotics. The sensor Nippon Signal FX6 has been used for autonomous person following [9] and for terrain classification [4]. A study of a continuous wave (CW) ToF sensor for mobile robot navigation is done by [7]. They explain the theory behind the sensors, and propose a model for error estimation and calibration. A study of the accuracy depending on the integration time of a CW ToF sensor is done by [14]. Chaibrando et al. [12] model the systematic error of the Swiss Ranger SR-4000 with a sinusoidal function. They also found the influence on the incidence angle of the object to be negligible. Different methods for calibration are presented in $[1,7,12,14]$. An investigation of influences on environment conditions like surface color, distances, speed and sunlight on sensors like the IFM O3D200 are done in [5]. Einramhof et al. compare two CW ToF sensors to an 2D laser scanner and a stereo vision system [15]. Structured light sensors and CW ToF sensors are well explained in the book of Dal Mutto et al. [2]. Quadratic noise models for structured light sensors have been proposed by $[6,16,17]$. There it is further mentioned, that the Microsoft Kinect cannot cope with direct sunlight and has serious problems with glass and black materials. But no detailed study is given. A standard camera calibration for the infrared camera is done by [6]. The error of fitting spheres is studied in $[3,8,17]$. It is found that the radius of the fitted sphere decreases with the distance to the sensor, but the incidence angle has no or little effect on the residues. The above mentioned publications mostly focus on benchmarking one sensor or one type of sensor.

A comparison of CW ToF sensors and the Microsoft Kinect to the ground truth of modeled data and a moving 2D laser scanner is done by Stoy et al. [10]. In their approach a 3D normal distribution transformation is used to estimate a true positive rate of each point cloud in a complex scene. Wong et al. [11] compare several 3D sensors, including a pan tilt laser scanner, structured light sensors, CW ToF sensors and stereo vision sensors. They use a static lab scene with a spatial checkerboard at the distance of $2 \mathrm{~m}$ to measure accuracy, precision and point density. However, we are not aware of a comparison of different 3D sensor types, i.e. 3D laser scanner, structured light and CW ToF, with a systematically variation of the conditions.

\section{Description of the Sensors}

The Nippon Signal FX6 sensor works similar to a 2D laser scanner, but instead of scanning a line, it scans an area. With one laser source and one photo diode, the time of flight of a laser pulse is measured. The manufacturer declares the maximum error as $80 \mathrm{~mm}(3 \sigma)$ at $5 \mathrm{~m} \mathrm{[13].}$

In contrast to the above mentioned principle, continuous wave (CW) ToF sensors, like the Swiss Ranger, IFM O3D200 or Fotonic E70P, use a strong light source that illuminates the whole scene with intensity modulated light. In 
a photo chip with demodulation pixels [7] the intensity, amplitude and phase shift of the reflected light are evaluated by a demodulation circuit in every pixel. Through the phase shift the distance of the reflected light can be determined. The repeatability of these sensors listed in the datasheets $[18,19]$ are $6 \sigma= \pm 8 \mathrm{~mm}$ for the IFM O3D200 and $1 \sigma= \pm 20 \mathrm{~mm}$ for the Fotonic E70P at a distance of $5 \mathrm{~m}$ and a target reflectivity of $90 \%$.

Sensors like the Microsoft Kinect and ASUS Xtion Pro Live project patterns of structured infrared light on the scene. Using an infrared camera the displacement of the pattern compared to a reference image can be determined. Through triangulation the depth of a pattern can be calculated using displacement [2]. As described in [17], through this technique the resolution and the random error $\sigma(d)$ deteriorate quadratically with the measured depth $d$.

$$
\sigma(d)=a+b d+c d^{2}
$$

The parameters $a, b$ and $c$ are found by measuring points on planes in different distances. Khoshelham et al. [6] propose for $a=b=0$ and $c=2.58 \mathrm{~mm} / \mathrm{m}^{2}$.

To compare the sensors in the state as they are delivered from the manufacturer no further calibration of the sensors is done in the following work.

\section{Experiments and Results}

The most important sources of errors for all sensors are the distance to the measured object as well as measured intensity and environmental illumination. Because the intensity is correlated with the background light, distance of the measured object, and surface of the object we try to change each parameter individually to evaluate its influence on the sensor.

In mobile robotics, for mapping, localization and obstacle classification, the accuracy is important, while for obstacle avoidance the accuracy of the perception plays a minor role but availability and reliability of the measured values are important. To evaluate the accuracy, we measure the normally distributed noise and the absolute error of the object size. For availability of the values we consider the rate of detected points. To have the same settings for every sensor and to be able to calculate the mean of the object position, the measurements are done in static scenes.

\subsection{Model of the Normal Distributed Noise}

Measuring physical properties is not possible without introducing uncertainty. Therefore many algorithms use probabilistic approaches to consider this uncertainty. If the uncertainty of the sensor readings is normally distributed, it is crucial to know the variance $\sigma^{2}$ of each measured value [20]. Since the noise of structured light sensors grows with the distance of the measured object [16], we experimentally estimated the standard deviation $\sigma$ of the sensor readings using objects at different distances and generated a model from these samples. 
The theoretical noise of structured light sensors can be modeled by

$$
\sigma_{d}=\frac{d^{2}}{b f} \sigma_{p}
$$

where $\sigma_{d}$ is the standard deviation of the sensor reading, $\sigma_{p}$ is the standard deviation of the measured disparity, $b$ is the baseline and $f$ is the focal length of the sensor [6]. However, this model neither respects any sensor internal calibrations nor the particular algorithms used by the sensors. Therefore, we used the more general model (1), which is based on (2), but covers our experimental results and was also proposed in [17]. Similar to the approach in [16] and [6], we assumed the sensor noise to be normally distributed with zero mean. We also assumed all errors to be independent of each other and that the sensor readings do not have a systematic error: $d^{\prime}=d+\mathcal{N}\left(0, \sigma^{2}(d)\right)$, where $d$ is the true distance and $d^{\prime}$ is the measured distance.

To sample the standard deviation, we placed the sensor at distances from $d_{0}=0.6 \mathrm{~m}$ to $d_{n}=9 \mathrm{~m}$ in front of a planar surface. The distances are measured using a measuring tape with a resolution of $1 \mathrm{~mm}$. We used a whiteboard with a dimension of $3 \mathrm{~m} \times 1 \mathrm{~m}$ and placed it in the center of the sensor's viewing area. Depending on the opening angle of the sensor, the planar surface did not fill the whole viewing range. Therefore, we manually marked the planar surface in the depth image. For each distance, we used 30 frames for the evaluation.

To estimate the plane position, we used the method of least squares to estimate the model of the plane based on the point cloud of one frame. The displacement of one point's depth value to this plane is then the error $e$ of the reading. In the next step, we evaluated the statistics of the errors with respect to their distance $d$ from the sensor. Therefore, the error values were, depending on $d$, sorted into bins of $20 \mathrm{~cm}$ width, if $d$ is smaller than $2.5 \mathrm{~m}$. For $d$ greater than $2.5 \mathrm{~m}$, the bin width was set to $100 \mathrm{~cm}$. For each bin we calculated the standard deviation $\sigma_{d}$ of the error:

$$
\sigma_{d}=\sqrt{\frac{1}{n} \sum_{i=0}^{n} e_{i}^{2}}
$$

To estimate a continuous model over the whole distance space, we fitted a second order polynomial function over the sampled standard deviations, by minimizing the following error:

$$
E(a, b, c)=\sum_{d=d_{0}}^{d_{n}}\left(\frac{\left(a+b d+c d^{2}\right)-\sigma_{d}}{\sigma_{d}} \cdot w_{d}\right)^{2},
$$

where $w_{d}$ is the width of a bin. The estimated standard deviations and the estimated models are shown in Fig. 1. For better display, the sensor names are abbreviated in the figures as follows: FX6, IFM, Fotonic, Kinect, Xtion. The coefficients of the estimated models can be found in table 1.

We found, that the sensors based on the time of flight approach had an error behavior which could not be covered by a polynomial function for near distances. 
Table 1. Models of the Normal Distributed Noise.

\begin{tabular}{lccc}
\hline & \multicolumn{2}{c}{ Model $\sigma(d)=a+b d+c d^{2}$} \\
Sensor & $\mathrm{a}\left[10^{-3} m\right] \mathrm{b}\left[10^{-3}\right] \mathrm{c}\left[\frac{10^{-3}}{m}\right]$ \\
\hline IFM O3D200 & 9.181 & -3.533 & 3.503 \\
Fotonic E70P & 1.284 & 0.871 & 1.280 \\
Microsoft Kinect & 7.152 & -6.750 & 3.296 \\
ASUS Xtion Pro Live & -3.396 & 5.392 & 1.134 \\
\hline
\end{tabular}

This is caused by oversaturated pixels of the sensor if the measured object is in a near distance. Since the distance for which the pixels start oversaturating highly depends on the surface, we did not consider according standard deviation samples for the model.

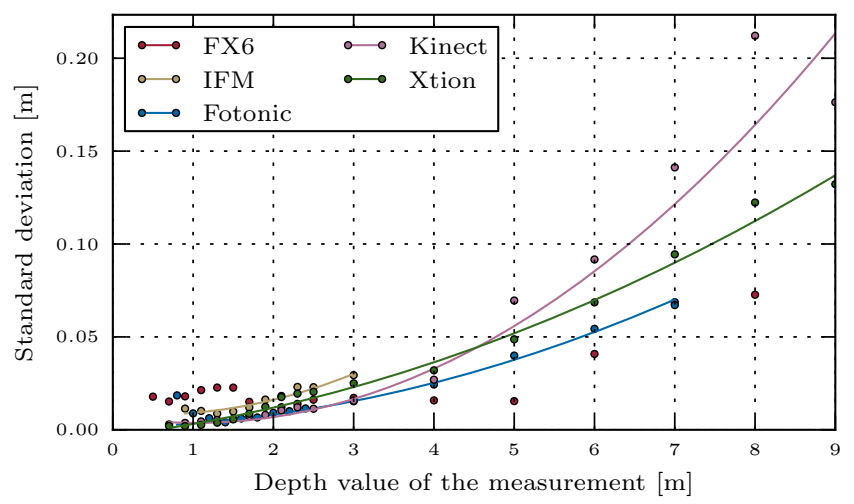

Fig. 1. Sampled standard deviations and modeled standard deviations of the different sensors.

Unlike the variance of all other sensors, the uncertainty of the Nippon Signal FX6 can not be modeled by a second order polynomial function of the distance. The main reason for this observation might be the measurement principle of the FX6 or sensor internal calibration methods. Therefore we do not provide a variance model of the Nippon Signal FX6. Also the range of the IFM O3D200 sensor was very limited. Therefore we estimated the variances of this sensor up to a distance of $3.5 \mathrm{~m}$. 


\subsection{Object Detection}

To evaluate the performance of object detection, we used a cylinder as sample object. For a cylinder it does not matter if it is seen from different sides and it is easy to fit in a model. In contrast to a cluttered scene, we know exactly the size and position of the object. This way the measurements can be repeated when new sensors are available. Compared to a sphere it is easier to change the surface by wrapping thin materials around a cylinder. Furthermore, cylindrical shapes e.g. legs, human body parts, wheels, buckets, stands, etc. are more common in real mobile robot environments. For the standard setup a cylindrical, orange plastic tube from a hardware store with radius $r=100 \mathrm{~mm}$ and height of $485 \mathrm{~mm}$ was placed in a dark room (2 Lux), one meter away from the sensor. The sensor was placed approximately $300 \mathrm{~mm}$ above the ground. From this setup the parameters were changed individually. At each setting 30 frames were recorded. The acquired point cloud is roughly cut in a way that only the cylinder and the ground plane are seen. The ground plane is detected by a RANSAC algorithm and then removed. From the leftover points the model parameters for a cylinder and the inliers were calculated using RANSAC algorithm with normals, as implemented in the Point Cloud Library [21]. The algorithm was set up with 1000 iterations and a threshold of $200 \mathrm{~mm}$. Finally the cylinder model was optimized by minimizing the error over all inliers. With the model parameters and the detected inliers, different evaluations were done to find three indicators.

The normally distributed noise is calculated from the standard deviation of the displacement using equation (3). To calculate the displacement $e_{i}$, the distance of a point to the cylinder axis of the model is calculated and the radius from the model is subtracted. Then the absolute error $E_{r}$ of the found radius $r_{f}$ to the true radius $r_{t}$ is calculated as $E_{r}=r_{t}-r_{f}$. Normally distributed noise and radius error indicate how accurate and reliable the measurements are. The rate of detected points $R$ is an indicator for the availability of sensor measurements under certain conditions:

$$
R=\frac{N_{d}}{N_{t}(\alpha, A, s)} .
$$

To be able to compare the number of detected points $N_{d}$ for different sensors and distances, this number was normalized by the number of theoretically visible points $N_{t}$. The number of theoretically visible points can be estimated using the angular resolution $\alpha$ of the sensor, which we get from the field of view and the number of pixels (table 2), and the visible area $A$ of the object at known distances $s$. For each sensor the three indicators in the standard setup are listed on the right of table 2 .

Change of Distance To evaluate the above described indicators for different depth values, the cylinder was placed at different distances in front of the sensor. The results for the three indicators for different distances are shown in Fig. 2. The top plot shows the normally distributed noise $\sigma$ as described above. The plot in the middle shows the mean radius error $E_{r}$ over 30 frames, the bars indicate the standard deviation of the error. At the bottom, the mean rate $R$ of detected 
Table 2. Given Sensor Properties and Experimental Evaluated Indicators

\begin{tabular}{lccccc}
\hline & Field of View [deg] Number of Pixels & \multicolumn{3}{c}{ Evaluated Indicators } \\
& $\mathrm{h} \times \mathrm{v}$ & $\mathrm{h} \times \mathrm{v}$ & $\sigma[\mathrm{mm}]$ & $E_{r}[\mathrm{~mm}] \mathrm{R}[\%]$ \\
\hline Nippon Signal FX6 & $50 \times 60$ & $59 \times 29$ & 10.8 & 51.1 & 14 \\
IFM O3D200 & $40 \times 30$ & $48 \times 64$ & 11.3 & 29.8 & 67 \\
Fotonic E70P & $70 \times 53$ & $160 \times 120$ & 5.0 & 19.6 & 87 \\
Microsoft Kinect & $57 \times 43$ & $640 \times 480$ & 1.9 & 0.9 & 84 \\
ASUS Xtion Pro Live & $58 \times 45$ & $640 \times 480$ & 1.7 & -2.5 & 88 \\
\hline
\end{tabular}

points and its standard deviation is plotted. It can be seen, that the normally distributed noise of the Nippon Signal FX6 increases between $0.5 \mathrm{~m}$ and $1.3 \mathrm{~m}$ but then stays almost constant around $10 \mathrm{~mm}$. At a distance greater than $2.5 \mathrm{~m}$ the cylinder in the 30 frames was not detected reliably enough to calculate the indicators.

For the IFM O3D200 the normally distributed noise also increases at close ranges but then decreases after $1.5 \mathrm{~m}$. The radius error of this sensor increases linearly with the distance at a rate of $4 \%$ and low standard deviation, while the point rate drops. For the Fotonic E70P the normally distributed noise increases linearly with a rate of approximately $3.4 \mathrm{~mm} / \mathrm{m}$. The radius error of the Fotonic is lower than the one of the IFM O3D200 but still increases linearly with a rate of $1.1 \%$. The rate of the detected points of the Fotonic E70P is low for distances less than $0.7 \mathrm{~m}$ but then reaches up to almost $90 \%$ and decreases linearly. The lower rate at a closer distance might be caused by oversaturation, when too much light of the modulated light source is reflected from the object to the sensor.

The sensors Microsoft Kinect and ASUS Xtion Pro Live are very similar. They have a low normally distributed noise, which increases linearly with a rate of $3.99 \mathrm{~mm} / \mathrm{m}$ and $3.88 \mathrm{~mm} / \mathrm{m}$. The mean radius error toggles around zero, while the rate of detected points decreases from $90 \%$ at $80 \mathrm{~cm}$ to $30 \%$ at $3.5 \mathrm{~m}$. Also some saturation effects are indicated by a lower rate at ranges closer than $0.7 \mathrm{~m}$.

The ToF sensors have a higher normally distributed noise and a higher radius error than the structured light sensors. To investigate if the decreased accuracy is caused by a lower number of points due to the lower resolution of the ToF sensors (as seen in table 2) two smaller cylinders with a radius of $55 \mathrm{~mm}$ and $80 \mathrm{~mm}$ are placed one meter apart from the sensor. It is found that the errors decrease with the radius. This means a small number of points does not necessarily increase the noise or the radius error. However, the higher radius error could be connected with the point rate.

Environmental Illumination Another big issue is environmental illumination. Therefore the cylinder was illuminated by different light sources with different illuminance (Fig. 3). The illuminance was measured with an external Lux meter placed next to the 3D sensor, pointing in the same direction. For lower illuminance, indirect daylight from a window and regular office illumination from 

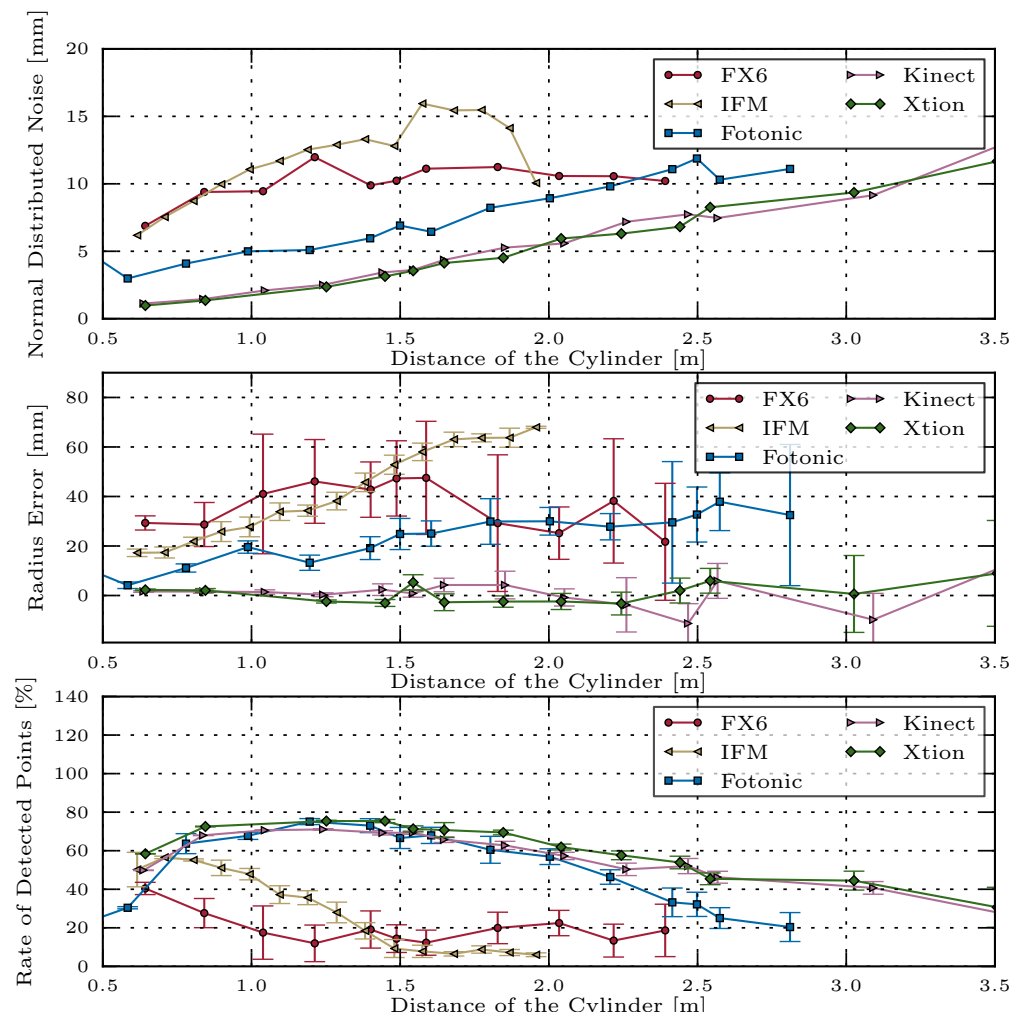

Fig. 2. The three indicators normally distributed noise (top), absolute radius error (middle) and rate of detected points (bottom) depending on the distance

a fluorescent lamp was used. For high illuminance a $575 \mathrm{~W}$ metal-halide lamp turned on in two steps was used as well as indirect and direct sunlight outdoors on a sunny day. In Fig. 3 the outdoor measurements are indicated through circles. It can be seen that for the Nippon Signal FX6 and the IFM O3D200 the lighting conditions have little influence on the noise, it increases a bit (15\%) for bright sunlight. This light has a strong effect on the Fotonic E70P, where the noise increases over $100 \%$. For the structured light sensors the noise stays almost constant until they do not deliver any measurements for illuminance higher than 4,000 Lux. It is hard to find a correlation between the radius error and the illuminance, except for the Fotonic E70P at 15,000 Lux. This is interesting, because the radius error gets a negative value of $-9 \mathrm{~mm}$ and a high standard deviation of $15 \mathrm{~mm}$. At this illumination the rate of detected points is also very low. That means that for directly illuminated scenes the Fotonic E70P has a similar noise and point rate as the other two ToF sensors but with a lower radius error. As it can be seen in the lower diagram of Fig. 3, indoor light does not affect the 
point rate of the structured light sensors, but outdoors with indirect sunlight the point rate can drop between $20 \%$ and $40 \%$.
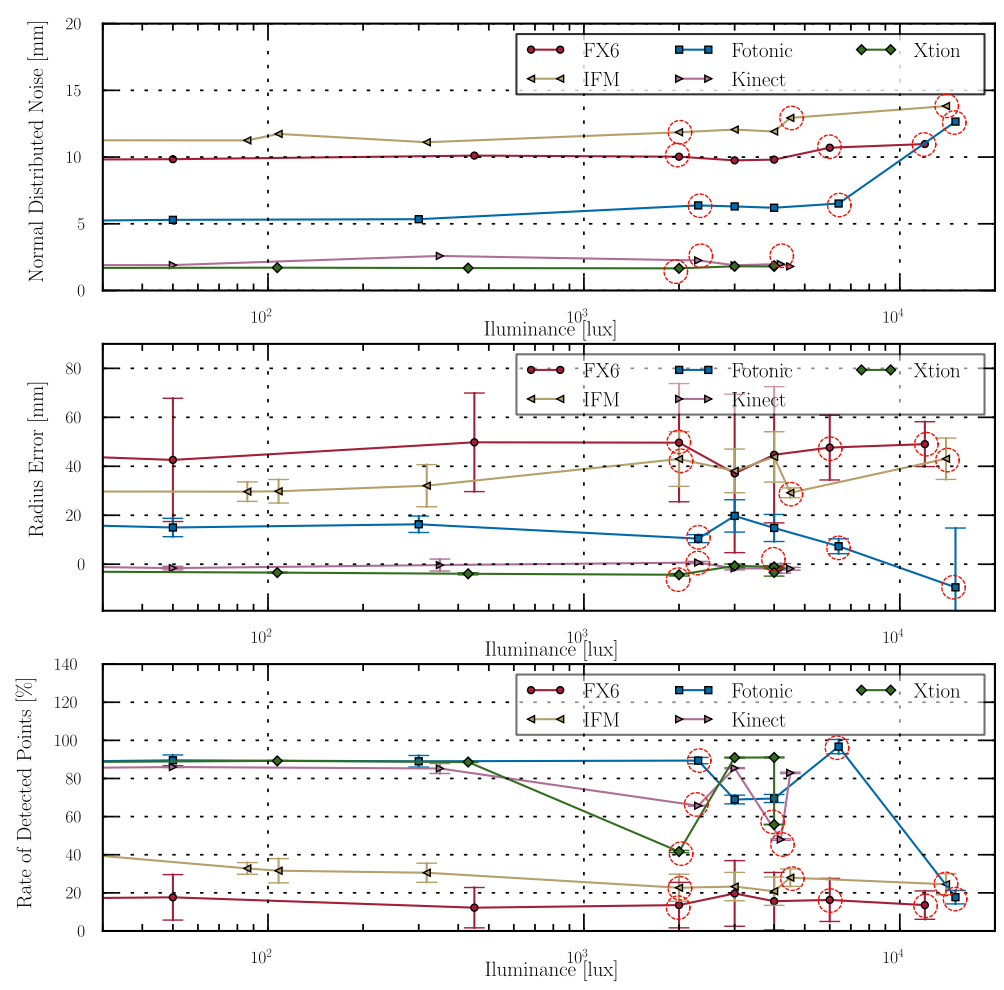

Fig. 3. The three indicators normally distributed noise (top), absolute radius error (middle) and rate of detected points (bottom) depending on environmental illumination.

Surface To evaluate the influence of the object surface the cylinder was covered with different materials. The materials, sorted from low to high reflectivity, are: black cotton cloth, dark jeans, brown package paper, white cotton cloth, white paper, the shiny orange plastic surface of the tube and shiny aluminum foil. As it can be seen in the top diagram of Fig. 4 the sensors Nippon Signal FX6 and IFM O3D200 have a high noise with dark surfaces and with shiny surfaces. The lowest noise occurs with white paper where the noise of the Nippon Signal FX6 is only $1.5 \mathrm{~mm}$ higher than the noise of the Fotonic E70P. Which is mostly constant around $4 \mathrm{~mm}$. It only increases with shiny surfaces, up to $9 \mathrm{~mm}$ for aluminum foil. For the structured light sensors the noise stays almost constant within less than $3 \mathrm{~mm}$ for all surfaces. The radius error also increases for shiny 
surfaces. For non shiny surfaces the radius error of the structured light sensors and the Fotonic E70P is negative. This means the found radius is greater than the true radius. The most negative value of $-18 \mathrm{~mm}$ appears for the Fotonic E70P with dark jeans. For the IFM O3D200 the most points are detected with the orange tube at one meter. The point rate of the Fotonic E70P is close to $100 \%$ for non shiny surfaces. For shiny objects the rate drops down to $10 \%$ with aluminum. Despite the lower rate of $40 \%$ at aluminum, the surface has no or very little influence on the noise and absolute error of structured light sensors. In the tested setups, aluminum is the worst surface for all sensors causing higher noise and radius error, as well as reducing the rate of detected points. Still a few points are detected at a distance of one meter and the structured light sensors are most robust in this case.

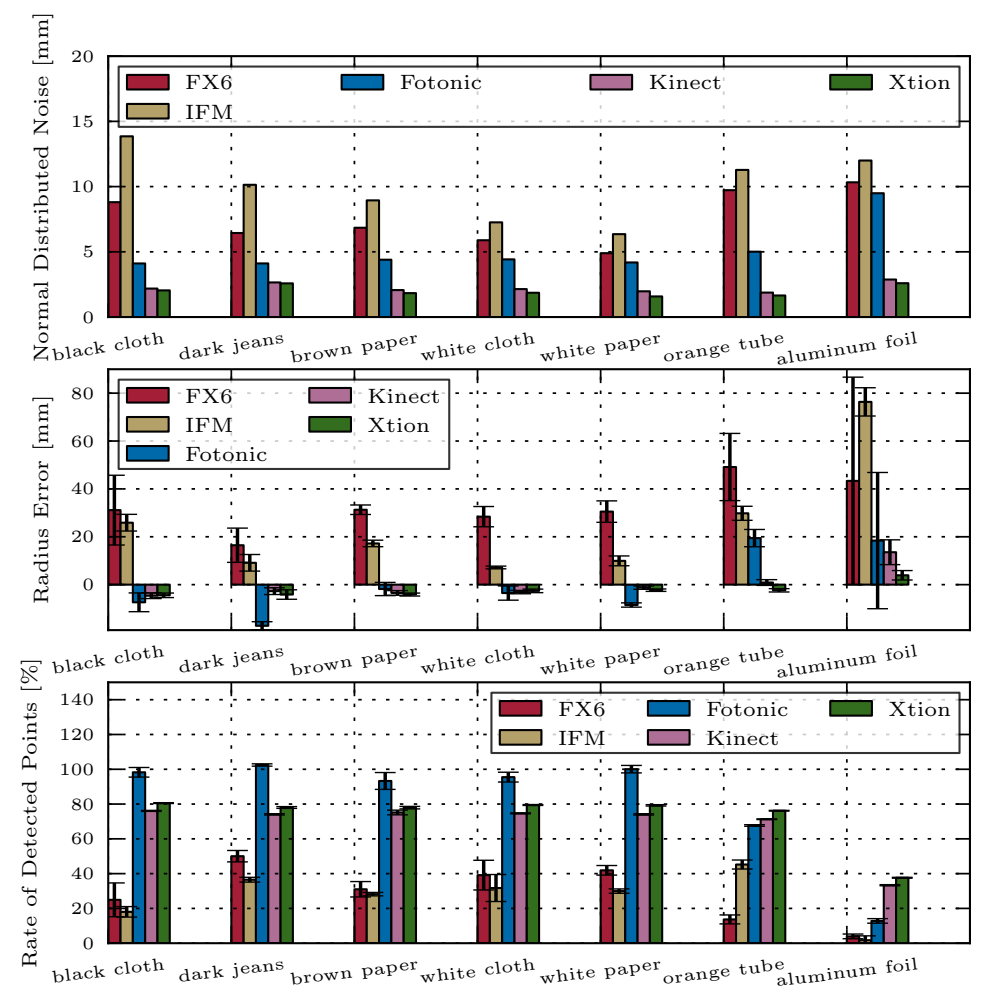

Fig. 4. The three indicators normally distributed noise (top), absolute radius error (middle) and rate of detected points (bottom) depending on the object surface. The surfaces are sorted from low reflectivity on the left to high reflectivity on the right. 


\section{Conclusion}

The paper presents an empirical comparison of several 3D sensors. For all sensors the same setups are used. This reveals the strength and weaknesses of the sensors under different conditions and enables an appropriate choice of a sensor for a certain application. For all sensors the normally distributed noise, the absolute radius error and the rate of detected points is evaluated under different conditions. The tested conditions are distance of the sensor to the object, environmental illumination and the surface of the object. It is found that for the most sensors, distance has the biggest influence. Further is found, that the absolute error of the radius increases with the distance for ToF Sensors. However, for the Nippon Signal FX6 the distance has not so much influence on noise and absolute radius error, even the environmental illumination has nearly no effect. This shows that this sensor is robust and reliable for most conditions. Still very shiny surfaces like aluminum have a huge influence on the error of ToF sensors where structured light sensors are more robust. The structured light sensors Microsoft Kinect and ASUS Xtion Pro Live are very accurate for close ranges up to $3.5 \mathrm{~m}$, where the increase of noise can be assumed to be linear. But for far ranges the noise increases quadratic, as expected from the sensors' working principle. Through this the ToF sensors have a lower noise at far distances. This is also found by [10]. Another drawback of the structured light sensors is that they do not deliver any measurements for direct sunlight on an object causing $\geq 15000$ Lux and the point rate drops already down between $20 \%$ and $40 \%$ with indirect sunlight at 2000 Lux. This makes these sensors not reliable for outdoor use. Having only investigated static scenes, we plan to compare the sensors for dynamic scenes.

\section{Acknowledgment}

This work is funded by the Germany Federal Ministry of Education and Research (BMBF Grant 01IM12005B). The authors are responsible for the content of this publication. Further, we thank Jan Leininger for assisting us with all the measurements.

\section{References}

1. D. D. Chow, Jacky C. K. and Lichti, A study of systematic errors in the PMD CamBoard nano," Proc. SPIE, vol. 8791, pp. 87910X-87910X-10, May 2013.

2. G. M. Mutto, Carlo Dal and Zanuttigh, Pietro and Cortelazzo, Time-of-Flight Cameras and Microsoft Kinect(TM). Springer Publishing Company, Incorporated, 2012.

3. J. Boehm, "Accuracy Investigation for Natural User Interface Sensors," in LowCost 3D - Sensoren, Algorithmen, Anwendungen, Berlin, Berlin, 2011.

4. S. Laible, Y. N. Khan, K. Bohlmann, and A. Zell, "3D LIDAR- and CameraBased Terrain Classification Under Different Lighting Conditions," in $A u$ tonomous Mobile Systems 2012, ser. Informatik aktuell. Springer Berlin Heidelberg, 2012, pp. 21-29. 
5. R. Klose, J. Penlington, and A. Ruckelshausen, "Usability study of 3D Timeof-Flight cameras for automatic plant phenotyping," Applied Sciences, vol. 69, pp. 93-105, 2009.

6. K. Khoshelham and S. O. Elberink, "Accuracy and resolution of Kinect depth data for indoor mapping applications." Sensors (Basel, Switzerland), vol. 12, no. 2, pp. 1437-54, Jan. 2012.

7. J. Weingarten, G. Gruener, and R. Siegwart, "A state-of-the-art 3D sensor for robot navigation," in IROS 2004 IEEE/RSJ International Conference on Intelligent Robots and Systems (IROS) (IEEE Cat. No.04CH37566), vol. 3, no. 2, 2004, pp. 2155-2160.

8. C. K. Molnár, B; Toth, "Accuracy Test of Microsoft Kinect for Human Morphologic Measurements," vol. XXXIX, no. September, pp. 543-547, 2012.

9. K. Bohlmann, A. Beck-greinwald, S. Buck, H. Marks, and A. Zell, "Autonomous Person Following with 3D LIDAR in Outdoor Environments," in 1st International Workshop on Perception for Mobile Robots Autonomy (PEMRA 2012), 2012.

10. T. Stoyanov, R. Mojtahedzadeh, H. Andreasson, and A. J. Lilienthal, "Comparative evaluation of range sensor accuracy for indoor mobile robotics and automated logistics applications," Robotics and Autonomous Systems, vol. 61, no. 10, pp. 1094-1105, 2013.

11. U. Wong, A. Morris, C. Lea, J. Lee, C. Whittaker, B. Garney, and R. Whittaker, "Comparative evaluation of range sensing technologies for underground void modeling," in Intelligent Robots and Systems (IROS), 2011 IEEE/RSJ International Conference on, Sept. 2011, pp. 3816-3823.

12. F. Chiabrando, R. Chiabrando, D. Piatti, and F. Rinaudo, "Sensors for 3D Imaging: Metric Evaluation and Calibration of a CCD/CMOS Time-of-Flight Camera," Sensors, vol. 9, no. 12, pp. 10 080-10 096, 2009.

13. P. Biber, U. Weiss, M. Dorna, and A. Albert, "Navigation System of the Autonomous Agricultural Robot "BoniRob"," cs.cmu.edu, 2010.

14. K. May, S. and Werner, B. and Surmann, H. and Pervolz, "3D time-of-flight cameras for mobile robotics," Intelligent Robots and Systems, 2006 IEEE/RSJ International Conference on, pp. 790-795, 2006.

15. S. O. Peter Einramhof and M. Vincze, "Experimental Evaluation of State of the Art 3D-Sensors," OGAM Annual Workshop of the Austrian Association for Pattern Recognition (OAGM 07), Krumbach; 05/2007; in: "Proceedings OAGMO7", (2007), 8 S., 2007.

16. S. A. Scherer, D. Dube, and A. Zell, "Using depth in visual simultaneous localisation and mapping," 2012 IEEE International Conference on Robotics and Automation, pp. 5216-5221, May 2012.

17. H. Gonzalez-Jorge, B. Riveiro, E. Vazquez-Fernandez, J. Martínez-Sánchez, and P. Arias, "Metrological evaluation of Microsoft Kinect and Asus Xtion sensors," Measurement, vol. 46, no. 6, pp. 1800-1806, July 2013.

18. Ifm, "O3D200 data sheet." [Online]. Available: http://www.ifm.com/products/us/ds/O3D200.htm

19. FOTONIC, "FOTONIC E40/70 data sheet," pp. 3-6. [Online]. Available: http://www.fotonic.com/assets/documents/fotonic_E40-70_high.pdf

20. S. Thrun, "Probabilistic Robotics," pp. 1999-2000, 2000.

21. R. B. Rusu and S. Cousins, "3D is here: Point Cloud Library (PCL)," 2011 IEEE International Conference on Robotics and Automation, pp. 1-4, May 2011. 